\section{A Systematic Review on \\ Blockchain in Education: \\ Opportunities and Challenges}

\section{Navin Duwadi}

\section{Abstract}

This study focuses on bloackchain as an emerging technology regarding its use to restructure the systems and advance education upon quality outcomes. What are the benefits of integrating blockchain in education concerning system reformation and advancement in developing better educational process for all encompassing learning outcomes? With the recent developments of blockchain applications across multiple domains, the education industry seems to be benefited from this technology in considerable degree. Transcripts and certificates play a vital role in individual's life so it needs to be stored in tamper-proof and long term available ledger. In pursuant to addressing afore stated question, this study bring information regarding what blockchain technology is, how it functions and what its uses if it comes to integrate with education at different levels. This may include reviewing the conceptual/knowledge bases, research and development studies and sources that introduce blcokchain including its features but with special highlight on how it has been integrated in the education sector especially in innovative situation.

To this end, this study was designed to provide a systematic review of all the literature focused on decentralized ledger technology. Multiple sources were reviewed following a systematic pattern that allows selection of relevant sources, sort out information being guided by the research question and make final selection of required information that connects with what and how of Blockchain in education.

Keywords: Blockchain, Education, Ledgers, Certificate, Decentralized technology

\section{Introduction}

Blockchain technology was proposed by someone called Satoshi Nakamoto in 2008 and was applied in a virtual currency application. Using blockchain technology, Nakamoto has invented a digital currency called bitcoin. It is a peer-to-peer network that uses distributed ledger technology to store and disseminate information (Nakamoto, 2009). After Satosh Nakamoto implemented blockchain technology to invent bitcoin there are many other cryptocurrencies also started emerging on the market.

1 Navin Duwadi is a faculty for graduate studies at Global College International and a director of Department of Information Technology.
Blockchain is a community record that consequently stores and checks transactions. Blockchain is a decentralized ledger of digital transactions. A blockchain is a long chain of connected data items that are saved on every participant device and can only be added by a highest number of participant those who agree. There are public blockchains that anybody may access and potentially contribute to, as well as private blockchains that are only used within a company or organization. The blockchain at the heart of the Bitcoin digital currency is the most well-known, but not the only, blockchain. The Digital ledger technology (DLT) powers Bitcoin, Ethereum, and other digital currencies. Use cases for a straightforward, obvious register of exchange information are various because DLT works through a decentralized stage making it tamper-proof transactions (Ark, 2018)

Every block in the blockchain can store a little volume of data (generally around $1 \mathrm{MB}$ ), which might be any information that has to be stored securely while still being widely disseminated. These might be information of monetary transactions (virtual currency) or exam credentials or learning records in the case of education (Sharples, \& Domingue, 2016). That data is kept on all participating devices and may be seen by anybody with the cryptographic 'public key,' but cannot changed or altered, even by the original author. The data records are timestamped, ensuring a reliable and accurate record of the new information.

The blockchain is an enormous and worldwide encoded data set that goes to the requiremen for decentralization and security of cycles normal to general people and organizations, finishing the syndication or monopoly of data by the elements that dealt with that data. In this sort of phenomenal and general arrangement, a group of individual records called "blocks" are joined in a "chain" that can't be altered, being approved by different PCs through the Internet. An enormous, decentralized, encoded, and open book of records where different specialists control the veracity of the information and guarantee its moral soundness were discussed. Its utilization incorporates the exchange of cryptographic forms of money, decentralized programming, and the enrollment of charges, and a long record of exchanges, everything being equal. (Gomez, 2021)

In the beginning, blockchain technology was limited only to the digital currencies application but these days there are lots of research undertaken to explore the importance of blockchain in other domains such as healthcare, education, smart cities, banking, insurance, and many more. The trend has shifted towards the security and verifiable system because blockchain provides an unbreakable and unhackable system for computer-based applications (Magazine, 2015).

The new and quick advancement of dispersed figuring and blockchain innovation has pushed us to reevaluate and reconsider a large number of the basic parts of the frameworks of education, literacy and training. Ideas like trust, worth, protection, and personality are for the most part, coming into question as another set-up of advancements has been introduced The recent and rapid advancement of distributed computing and blockchain technologies has compelled to reconsider and redesign many of our established educational institutions. A new set of technologies, concepts like trust, value, privacy, and identity are all being called into question when the blockchain technology is brought into the academic institution. 
The main concern of blockchain technology in the education sector is how this technology can provide the secure as well as a trustable and traceable exchange of educational information. Later that information is shared among the multiple institutions in the field of education including teaching and learning process that too encompass accreditation and validation of educational achievements that are in turn secured in the ledger (Blockchain in education, 2021). It has been found that Blockchain technology provides an environment where individuals can be the custodian of their academic records and won't necessarily require any intermediaries to record or alter records. Issuance, validation, and sharing of certificates can also be done by education providers using blockchain technology, a cryptographic data exchange which will ultimately help to prevent the certificate frauds that are seen so often.

A revolutionary field for technologies is identified in the education sector, an emerging area where the requirement for the certificate is fundamental. It can be found on the multiple research done by researchers, certain percentage of the applicants falsified the degree certificates. The need to check this sort of data is presently not accidental and might be valuable to keep away from certain ensuing debates (Sharma, 2018).

Students' records are a very crucial part of the education sector, and with blockchain innovation, resources like learner participation, courses, and installments toward educational cost on the off chance that they go to a public or private school, grades, coursework, and surprisingly their certificate can turn out to be essential for their own blockchain record. Since these records can't be erased, this assists with information security in that it is permanent Student records are the property of the students rather than to the school. With blockchain, no member can alter a record after it has been saved. On the unusual chance that a record has a blunder, another record should be added to address it, and both the erroneous and right records will stay apparent (Hance et al., 2021).

\subsection{Problem statement}

Document forgery is a developing issue that requires immediate attention. In recent years, the fake replication of certificates has expanded dramatically, posing a threat to education policy stakeholders. Fake diplomas not only deceive companies and colleges, but they also deceive students. These days' education institution and workforce seems to have victim of the fraudulent academic documents. It is often found that some people have faked their document to enter into the professional career. It has a negative impact on the society because one without required qualification can damage the work force and the reputation of the organization where they are working. It was found that even some reputed companies have similar kind of issues on the past. In the context of developing countries like Nepal, people are working in the sensitive areas such as medical industry and academic institution with the fake qualification that will eventually destroys life of the pupils. So, the immediate action is needed to control the spread of the forgery documents and blockchain based verification system will be able to address the issue.

1.2 Objective

The main goal of our research is to,

(ii) classify blockchain-related existing research,

(iii) identify the opportunities and challenges of blockchain technology implementation in education, and

(iv) map the connections between the discovered blockchain opportunities and challenges with the identified research topics.

The blockchain simply refers to the data records that have been hosted on decentralized networks and are a part of the blockchain and cannot be modified. Blockchain is a peerto-peer network that uses cryptography technology to keep data safe. In the blockchain, all existing records are managed by a group of nodes that cannot be owned by a single entity so updating or even damaging records on all networks is nearly impossible. All of the transactions that the block will bring are linked together so that they may be validated and signed using cryptographic evidence that the blockchain and its volunteers have solved

The application of blockchain-like virtual currency such as Bitcoin uses the public key infrastructure (PKI) mechanism. In PKI, generally, pair of a public and private key is distributed to the users. The public key is used to identify the address of the currency wallet and a private key is used to identify the authenticity of the user. The ledger of the transaction consists of the sender's and receiver's public key and the value of the transaction. In about ten minutes, the transaction will be written in a block (Yli-Huumo et al., 2016). A previously written block is then linked to this new block. All blocks, including information about each transaction, are saved in the users' disk storage, which is referred to as nodes. Each node stores information about all Bitcoin network transactions and uses prior blocks to verify the integrity of each new transaction. Checking the accuracy of transactions rewards the nodes (Haugsbakken \& Langseth, 2019). Mining is the term for this process, and it is supported by Proof-of-Work, which is one of the key concepts in Blockchain technology. A consensus exists amongst all nodes when all transactions are successfully confirmed (Yli-Huumo et al continuous chain. This chain of blocks is the public ledger technique of Blockchain.

According to Swan (2015) Blockchain is the decentralized overseeing strategy, intended for distributing and moving cash for the clients of the digital currency. This strategy can support the public record of all virtual currencies transaction that have at any point been executed, with no control of a third-party association. The benefit of Blockchain is that the 
public record can't be adjusted or erased after the information has been supported by all nodes. This is the reason Blockchain is well known of its information trustworthiness and security attributes (Casino et al., 2019). Blockchain innovation can likewise be applied to different sorts of users. It can, for instance establish an environment for virtual contracts and peer to peer information sharing in a cloud computing service (Swan, 2015). The solid place of Blockchain strategy, information trustworthiness, is the motivation behind why its utilization stretches out additionally to different administrations and applications such as an education sector.

\subsection{Blockchain key features}

\subsubsection{Corruption proof transaction}

There are several attractive blockchain qualities, but corruption proof is probably one of the most important features of blockchain technology. Corruption proof also called Immutability refers to the inability to change or alter anything (Iredale, 2021). This is one of the most important blockchain aspects for ensuring that the technology stays as it is - a permanent, unchangeable network. Blockchain technology differs from traditional financial systems in a few ways. It ensures the blockchain features through a collection of nodes rather than depending on centralized authorities (Haugsbakken \& Langseth, 2019). The digital ledger is duplicated on every node in the system. To add a transaction, each node must first verify its legitimacy. If the majority agrees that it is correct, it is entered into the ledger. This increases transparency and makes it impenetrable to corruption (Iredale,2021).

As a result, no one can add any transaction blocks to the ledger without the approval of the majority of nodes. Furthermore, once the transaction blocks add to the ledger no one can go back and alter the transaction. Thus, not any user will be able to change or delete the transaction added on the ledger.

\subsubsection{Decentralized technology}

The network is decentralized, which means there is no governing authority or one individua in charge of the network. The network is instead maintained by a collection of nodes, making it decentralized. This is one of the most effective aspects of blockchain technology. The blockchain puts the users, in a simple position. Users can access the system straight from the web and keep the assets there because it does not require any regulating authority. Anything from bitcoins to crucial documents, contracts, and other significant digital assets can be stored. And, thanks to blockchain, the user will be able to exert direct control over them by utilizing the private key (Iredale, 2021).

\subsubsection{Improved security}

Cryptography, when combined with decentralization, adds another degree of security for individuals. Cryptography is a complicated mathematical procedure that protects against attacks. Every piece of data on the blockchain is cryptographically hashed. In simple terms, the network information conceals the underlying nature of the data (Iredale, 2021). Any input data is passed through a mathematical method that generates a different form of value, but the length remains constant. Each block in the ledger has its own hash and also contains the hash of the previous block. Users will be provided with a private key to access the data and a public key to make the transaction (Daley, 2021).

\subsubsection{Distributed ledgers}

In terms of hierarchy, all full nodes on the blockchain network are equal, and each node behaves as an autonomous agent. On the local space, they store and maintain their own subsets of the shared ledger. This kind of decentralized storage can solve a lot of the issues that a centralized system has. It is frequently utilized in the field of blockchain because it can improve the system's reliability, availability, and access efficiency (Raman \& Varshney, 2018).

\subsubsection{Consensus mechanism}

Blockchain is a type of distributed ledger. Each and every user has the right to store and manage the database and all the users will have a copy of the ledger. A consensus mechanism refers to a set of rules that ensure all participants are on the same page of the ledger, thus ensuring that the blockchain information stored in each node is not falsified. Nowadays, there are many consensus mechanisms available such as proof of stake (PoS), proof of work (PoW), and delegated proof of stake (DPoS) (Mingxiao et al., 2017).

\subsubsection{Quick settlement}

Transaction in a traditional system takes a long time to settle down. Blockchain provides a faster settle downtime for the transaction (Arndt, 2019). Comparing multiple applications of blockchain such as virtual currencies and digital certificates in education. People travel overseas for higher education and it will be very hectic for them to receive documents in a traditional way. Where Blockchains are now far too quick for them, and they can easily transmit money or receive documents using blockchain technology (Iredale, 2021). The smart contract system is another interesting fact. This may make it possible to conclude any type of transaction more quickly. To this day, this is one of the most valuable blockchain characteristics. People can send and receive money or documents for a little cost now that the third party is out of the way.

\subsection{The benefits of blockchain in education}

\subsubsection{Enhanced transparency}

The immutable ledger technology of blockchain produces a chronological record of realtime events. This is excellent for validating transcripts, displaying a complete report card, and keeping students up to date on their progress. When a student submits their assignment via blockchain, they can't "lose" it or claim it was misplaced by the teacher (Daley, 2021). 


\subsubsection{Accountability through digital contracts}

Smart contracts will soon be available to teachers, university officials, and students. Students and teachers, for example, could sign a digital agreement outlining the limitations of an assignment, as well as the due date and grading deadline. Smart contracts can potentially be used to pay off student loans (Daley, 2021)

\subsubsection{Incentivization of learning}

Tokenization has become a cornerstone of blockchain technology. Academic institutions will soon be able to incentivize students to pay off their student debts on time, and professors will be able to motivate students by rewarding them with cryptocurrencies if they perform well or complete a specific major. Tokenization's gamification aspect of teaching has proven to be extremely effective (Daley, 2021).

\subsubsection{Learning performance and personalization}

Educators now have additional resources to monitor and oversee their pupils' performance because to the transparency provided by blockchain data storage. Schools may provide a fully tailored learning environment by employing smart contracts and their output as tools to determine where a student is lagging. Institutions across the school district can access student performance data stored on a blockchain and utilize it to improve educational processes (Pixelplex, 2021).

\section{Research Methodology}

This study follows systematic literature review. Systematic literature review is a method of collecting, assessing, and interpreting all relevant research for a certain research question, topic area, or phenomenon of interest. Primary studies are individual studies that contribute to a systematic review; a systematic review is a type of secondary study. A systematic literature review was conducted by searching for relevant papers according to Petersen et al. (2008) principles. The systematic review process was chosen as research methodology because the goal of the review was to explore the existing studies related to Blockchain technology. The following subsections go over the steps of the guidelines.

The overall methodological approach includes the following,

Identify the need for a review, specify the research questions and develop a review protocol.

2) Identify the research, select the studies, assess the quality, take notes and extract data, synthesize the data.

3) Carried out the survey applications with blockchain in use for academic purposes

\subsection{Results of the review}

The need for this review paper is to address the research trends as well as opportunities and challenges in the blockchain and education sector. By looking at the existing secondary research, it is clear that there are several gaps that has not yet been addressed and that need to be addressed in order to have more practical implications. This paper has followed the qualitative research methodology to explore existing research and identify the gaps. To address the gap this paper has identified, gathered and interpreted the research article to answer the specific research questions, and followed the systematic pattern to develop review protocol by conducting research, separating multiple paper with certain criteria.

\subsection{Research question}

The study seeks to answer these research questions that are analytical in nature:

1) When and how often were blockchain research articles for education published?

The main aim of the research question is to identify the trends that includes the publication trends over the time in a multiple region and also helps to understand the research topic, blockchain in education is accepted by scientific research community as a relevant research topic.

2) What are the opportunities that blockchain technology could bring into the field of education?

This research question intends to identify the opportunities of employing blockchain technology for education that have been discovered in the peer-reviewed literature. It will also aid future scholars in their efforts to enhance blockchain adoption in the realm of education.

3) What are the various challenges while implementing blockchain technology in education? This research question intends to identify the challenges of implementing blockchain technology for education that have been found from the peer-reviewed literatures. It will also help future scholar to improve blockchain adoption in educational institution.

\subsection{Conducted search}

A comprehensive literature search was conducted between August 2021 to address our core research question, and the results were updated in September 2021. Scopus, IEEE Explorer, ACM Digital library and Google scholar were utilized as the main scientific database, and all paper titles were searched for the term blockchain, decentralized ledger, education and distributed ledger, and performed different combinations of keywords to search over the search engines. Additional searches were carried out using the referenced works of relevant articles. At the end of the process we have found around 300 publication in multiple databases. 


\subsection{Inclusion and exclusion criteria}

3.4.1 Inclusion criteria

Paper that contains present application of blockchain technology in education field.

Peer-reviewed research articles, conference proceedings papers, book chapters, review papers, short surveys, serials etc. that present information about blockchain technology in education.

Full paper available on the internet related to the blockchain technology in multiple domains

\subsubsection{Exclusion criteria}

Paper that are not written in English, paper without missing abstracts, notes and editorials.

Software oriented articles of the blockchain technology

\section{Discussion and Findings}

The results of the systematic literature review are discussed in this section. Based on the review most the research paper has been analyzed from the online database. Below is an in-depth review of our study's main findings in relation to each of the research questions.

\subsection{Example of blockchain based educational service providers}

\subsubsection{Blockcerts}

Blockcerts is a Blockchain community based project of MIT based on Cambridge, Massachusetts is an open standard that allows users to create, issue, examine, and verify blockchain-based certificates. These digital recordings are cryptographically signed, tamper-proof, and shareable and are stored on a blockchain (Daley, 2021). The goal is to inspire a wave of innovation that will allow people to own and share their own official records. Academic achievements, grades, transcripts, and even degrees can all be saved on the Blockcerts, allowing immutable access to prior academic history. The main feature of the Blockcert is to store student's digital credentials and offers multiple applications such as learner's application, teacher's application and user verification application.

\subsubsection{APPII}

APPII based on London, England used to authenticate credentials using blockchain technology. To validate the academic credentials of prospective students and lecturers, the company uses blockchain, smart contracts, and machine learning. Users of APPII build a profile and complete their academic $\mathrm{CV}$, which includes their educational history and transcripts. APPII then uses blockchain to validate a user's identity and save their data in its Blockchain (Daley, 2021). APPII has created a system that registers and evaluates the credentials and experience of pupils and those already in the workforce utilizing modern technologies such as blockchain and smart contracts. APPII also can be used as a career verification tool that helps screening candidate resume for further selection with the help of the blockchain technology.

\subsubsection{Gilgamesh platform}

Gilgamesh is a social platform based on San Jose, California for information exchange that uses Ethereum smart contracts and blockchain technology. The network changes how readers, critics, and authors interact and communicate with one another. Users can gain and transmit knowledge in a secure environment using the Gilgamesh platform, which supports widespread learning and education and urges the human race to become more thinking and information-centric (Tuson, 2018). Using the feature of the blockchain technology Gilgamesh platform has provided secure and reliable platform for learners and educators to share common platform for certificate validation and verification.

\subsubsection{ODEM}

ODEM is a blockchain-based educational online platform that provides students with economical academic courses that can be tailored to their specific needs (Pixelplex, 2021). Students can communicate directly with academic experts and agree on the optimum learning environment. The platform employs smart contracts and blockchain-enabled payment systems to incentivize and reward students for their academic endeavors, as well as to encourage teachers to produce better courses. The blockchain stores all of the certifications that students have earned. As a result, they may be easily accessible and shared on social media, as well as used as evidence in digital CVs.

\subsubsection{Sony Global Education}

Sony Global Education based on Tokyo, Japan is a distributed ledger that allows various education providers to share their students' results (SGE, n.d). The blockchain network serves as a repository for each student's lifetime digital transcript, which can then be used for university transfers and education verification at the workplace. Sony plans to expand the platform and create the foundation for a next-generation educational ecosystem in which professors and students cooperate and share digital currency rewards.

\subsubsection{Blockchain Education Network}

The Blockchain Education Network (BEN) based on Newark, New York formally known as College Cryptocurrency Network (CCN) is a global non-profit organization dedicated to building a strong network of student groups for blockchain in education, invention, and expansion (Ullah et al., 2021).

BEN was created to assist emerging, growing, and not-yet-existing college organizations in establishing a presence on their campuses, receiving administrative recognition and financing, and connecting students with similar college and institutions. In order to change the face of electronic finance and innovation, BEN provides educational and professional materials, contacts, speakers, and guidance (BEN, n.d). 


\subsubsection{Disciplina}

Disciplina based on Kesklinna, Tallinn, Estonia is a personalized learning management system that keeps track of learners' academic achievement using blockchain technology (Daley, 2021). Each student is given a score based on their academic performance, extracurricular activities engagement, and other accomplishments. These results are used by schools and institutions that have registered on the platform to personalize the learning process and generate unique programs for each course. Disciplina also attempts to improve communication between students and teachers by providing easy-to-use tools for grading, rating, and reviewing. Recruitment firms and businesses can also use the platform to find potential employees (Pixelplex, 2021).

\subsubsection{Parchment}

Parchment based on Scottsdale, Arizona, provides digital credential services to students, academic institutions, and companies, and uses blockchain in education. K-12 educators can upload any significant developmental progress to the company's blockchain. The platform is used by higher education institutions to assess academic merit, process applications, and issue unchangeable diplomas. Students also have 24/7 access to all educational information and can simply share their academic achievements with potential employers and educational institution (Daley, 2021)

\subsubsection{Bitdegree}

BitDegree based on Kaunas, Lithuania is more tech-focused online education platform integrates blockchain ledgers and tokenization. To help more people learn about digital ledger technology and eventually adopt a blockchain-related career, the organization offers online courses such as "Cryptocurrency for Dummies: Ethereum vs Bitcoin and Much More." The site also encourages education by awarding tokenized scholarships for completion of the course or fulfillment of specified goals (Daley, 2021)

\subsection{Applications of blockchain technology in education}

Some universities and institutes have begun to incorporate blockchain technology into their educational programs, with the majority of them using it to assist academic degree management and summative assessment of learning outcomes (Chen et al., 2018). The first category, Certificate Verification, included research on the use of blockchain technology to create digital certificates that can be consulted and verified by third parties via an untrustworthy and immutable proof system, or even revoked if they were issued incorrectly. Fake degrees, diplomas, and certificates are a serious issue in the educational area these days, and verifying their authenticity takes a lot of time and effort. Blockchain technology has the potential to help reduce certificate and degree fraud. As a result, a number of educationa institutions have begun to employ this technology to develop a digital certificate that can be consulted and validated by third parties via an unreliable and unchangeable proof system. (Loukil et al., 2021).
The second category, Student-centric Educational Record Management, included a variety of topics. Students should be able to access and control their own educational data via blockchain technology. For example, Liang and Zhao (Liang and Zhao, 2020) developed a blockchain-based system for full quality assessment of students to overcome issues with keeping students' study data

Furthermore, Blockchain can also function as a skilled based transformation bank. In particular, the blockchain learning ledger keeps track of the users' knowledge and skills growth by recording extensive information about their learning experiences. All of them can be converted into a type of digital currency and stored on a blockchain network in accordance with a set of detailed standards. Students will be rewarded for their academic efforts, which is also known as earning through learning. (Chen et al., 2018)

Another application area, Blockchain-based Educational Institute Systems, contained research on blockchain applications for transferring academic credits between universities. According to the articles analyzed, the blockchain may be utilized to efficiently share information without the involvement of third parties or intermediaries. (Loukil et al., 2021).

\subsection{Opportunities of integrating blockchain technology in education}

Many blockchain projects from various areas share the goal of reducing paper-based workflows, lowering administrative expenses, and improving the efficiency of routine procedures involving multiple parties. Arndt (2019) explains the blockchain is a technology that allows for the creation of a tamper-proof record that is shared by a number of stakeholders who may have competing interests and hence do not trust each other as the sole source of information. As a result, various stakeholders can use blockchain systems to establish safe and trustworthy records. Furthermore, by merging blockchain and smart contracts, numerous operations involving several stakeholders may be reliably automated. Data stored by smart contracts may be traced in this way, holding parties accountable and resulting in a highly transparent system that can be easily validated by third parties (GarciaFont, 2020). By implementing the blockchain technology in education, every document can be shared through digital media and reduces the administrative cost and creates the paperless environment.

According to Loukil et al., (2021) adopting the blockchain to secure and verify shared evidence can improve the education area. Because student records are saved on a blockchain, papers such as diplomas and certificates can be eternally secured and certified, even if the institutions that issued them closed down or the entire educational system collapsed due to conflicts or natural disasters. Because they can verify the validity of their certificates issued to third parties directly on the blockchain, the nature of blockchain technology can help educational institutions save time and resources associated with transactions to confirm the validity of their certificates issued to third parties.

Some of the reviewed articles has explained that many blockchains also support smart contracts. This means that lectures and courses can be written into the blockchain an 
run automatically when certain criteria are satisfied. A teacher, for example, could assign assignments to students. The smart contracts on the blockchain may verify the accomplishment of each task automatically. Teachers might be paid in crypto tokens for completing all responsibilities, and pupils could be given credits. This method might be used to lay out entire courses. (Cedeño et al., 2020)

Students, teachers, professors, and researchers produce high-quality content on a regular basis. However, getting published is a difficult task. Science has a long history of valuable research that has been rejected by scientific publications for a variety of reasons: however, publishing on a blockchain could enable new writers, researchers, and others break into the business. Blockchains may also aid in the administration of rights and the prevention of piracy. Students and teachers who write stories, make music, documentaries, or other short films could share their work on the blockchain. This would not only save time in finding a publisher, but it would also defend against piracy, enhance copyright management, and reward the author directly based on how popular or well-rated their creations are. (Cedeño et al., 2020)

Blockchain technology also provides opportunity to create better learning platform in educational institutions. The creation of improved online learning platforms will benefit from blockchain technology. Universities, in particular, can create useful education systems and programs that connect students and teachers. Schools use the education idea to improve access to and exchange of study resources. Users purchase internal tokens in order to request feedback from on-call online tutors. They can also access educational services and download learning materials. (NuWire, 2021)

Tokens are earned when students on educational platforms view tutorials and invite new members to the system. When people connect with their material, content providers earn extra tokens.

\subsection{Challenges of integrating blockchain technology in education}

This challenge invited teams to articulate a vision and design pilots that empowered all learners, unlocked lifelong learning, and improved economic mobility. In doing so, the teams addressed various important characteristics of an ecosystem-first design approach:

\section{- Scalability}

The amount of transactions in blockchain technology is increasing every day and making the distributed ledger bulky. In the blockchain, every node should store transaction records and information (Zheng et al., 2017). With the increasing number of blockchain nodes in the network, it reduces the efficiency of the algorithms and the response time of the nodes. Although it has been said that blockchain technology is tamper-proof but still there are a lo of cases where hackers have attacked the system so this challenge is not addressed properly there will be a high risk of the leakage of teachers and student information. (Ma \& Fang, 2020)

\section{- Interoperability and open design}

These days many organizations and industries are adopting blockchain technology and there is a trend that they develop their systems with different characteristics. Unfortunately, there is no universal standard to activate separate networks to communicate with each other in blockchain technology. Blockchain interoperability refers to the capability to share and access information across the network. This could be a challenge of blockchain technology in the field of education where student's sensitive data will be shared across the network (Malavolta et al., 2019)

\section{- Non-technological factors}

\section{Blockchain engineers and experts}

Blockchain technology is a new and emerging field and it can be found that there is still a lack of skilled professionals, engineers, and experts on the market. With the rapid development of blockchain applications in multiple domains, the demand for human resources of blockchain technology is increasing (Ma \& Fang, 2020). Most of the companies, either start-up or well established are looking for blockchain developers to implement blockchain-based systems. Nevertheless, the education field is sensitive so high-level research and development group is needed to develop and implement blockchain technology in the higher education sector. Because of the increasing demand on the market place blockchain professionals are very expensive to afford for the educational institution. Skilled professionals will be a huge challenge for an educational institution to implement blockchain technology.

\section{- Lack of policy protection and practical experience}

The majority of blockchain research now focuses on disrupting the existing finance sector, and its use in education is very new (Liang \& Zhao, 2020). Furthermore, lack of policy protection and relevant skills provide barriers to progress. First, decentralization will have a significant impact on traditional educational institutions due to a lack of legislative protection and supervision. The traditional education platform incurred significant losses, leading to a hostile attitude toward innovative applications among important institution and departments. Second, due to the limited number of application instances, professionals and researchers are waiting to see what happens next and lack the drive to promote new applications. (Chen et al., 2018)

\section{- Trust factors}

The trust factor is one of the major obstacles among blockchain users to implement blockchain technology. Especially in the education sector trust plays a vital role to keep student's and teacher's information securely over the blockchain network. There are two different types of challenges related to trust. Firstly, the organizations may not be able to trust the technology and may not trust the other organization on a distributed ledger network. (Ma \& Fang, 2020) 


\section{- Cost}

Another important factor for blockchain's poor adoption in the education sector is that few decision-makers in the field have a good understanding of what it is and how it may improve the entire system. The main reason will be costs: the cost of altering the environment, setting up the infrastructure, the cost of blockchain development, and the cost of blockchain training for employees. It may be difficult to predict the long-term financial benefits of blockchain integration, which include lower administration and record-keeping costs, as well as additional gains from implementing cryptocurrency payments and rewards (Pixelplex, 2021).

\section{Conclusion}

The use of blockchain technology in education is still in its initial phase. As a result, a review of current blockchain research in the field of education is required to identify specific research gaps that should be addressed in future research. The goal of this research was to give a thorough examination of blockchain applications in the sector of education. We conducted a systematic literature review for this purpose. We were able to identify the opportunities and challenges of implementing blockchain technology into education because of our review of literatures. As a result, this analysis can help future research efforts in the field of blockchain adoption in education overcome present solution limitations. Although this technology is still in its early stages of development and must undergo testing, it has the potential to spur a lot more innovation in the future. In our future work, we will introduce tokenization of educational activity in detail. Furthermore, monetizing all of the students activities on blockchain network will inspire students to participate in multiple activities such as sports, attendance, and homework and so on.

\section{References}

Arndt, T. (2019). An overview of blockchain for higher education. Proceedings of the 11th International Join Conference on Knowledge Discovery, Knowledge Engineering and Knowledge Management. https://doi. org/10.5220/0008343902310235

Ark, T. V. (2018). 20 ways blockchain will Transform (Okay, May IMPROVE) EDUCATION. Forbes. https:// www.forbes.com/sites/tomvanderark/2018/08/20/26-ways-blockchain-will-transform-ok-may-improveeducation/?sh=cb87fff4ac91.

Blockcerts. (n.d.). Blockchain credentials. Blockcerts. Retrieved September 27, 2021, from https://www.blockcerts. org/about.html.

Blockchainedu.org. (n.d.). The blockchain Education NETWORK (BEN). Retrieved September 28, 2021, from https://www.blockchainedu.org/about.

Brown, M. (2021). 5 challenges with blockchain adoption and how to avoid them. SearchCIO. https://searchcio. techtarget.com/tip/5-challenges-with-blockchain-adoption-and-how-to-avoid-them.

Casino, F., Dasaklis, T. K., \& Patsakis, C. (2019). A systematic literature review of blockchain-based applications: Current status, classification and open issues. Telematics and Informatics, 36, 55-81. https://doi.org/10.1016/j. tele.2018.11.006

Cedeño, J. (2020). How blockchain could impact education in 2020 and beyond. Getting Smart. Retrieved September 30, 2021, from https://www.gettingsmart.com/2020/02/23/how-blockchain-could-impact-education-in-2020and-beyond/.

Chen, G., Xu, B., Lu, M., \& Chen, N.-S. (2018). Exploring blockchain technology and its potential applications for Education. Smart Learning Environments, 5(1). https://doi.org/10.1186/s40561-017-0050-x

Daley, S. (2021.). 9 blockchain education companies Earning Straight A's. Built In. Retrieved September 27, 2021 from https://builtin.com/blockchain/blockchain-education.

Garcia-Font, V. (2020). Blockchain: Opportunities and Challenges in the Educational Context. Lecture Notes on Data Engineering and Communications Technologies. https://doi.org/10.1007/978-3-030-29326-0

Gomez, D. (2021). Blockchain in education, a large and global encrypted database. eLearn Center Blog. https:// elearncenter.blogs.uoc.edu/blockchain-in-education/.

Guustaaf, E., Rahardja, U., Aini, Q., Maharani, H. W., \& Santoso, N. A. (2021). Blockchain-Based Educatio Project. Aptisi Transactions on Management (ATM), 5(1), 46-61. https://doi.org/10.33050/atm.v5i1.1433

Hance, M. (2021). What is blockchain and how can it be used in education? MDR. https://mdreducation. com/2018/08/20/blockchain-education

Haugsbakken, H., \& Langseth, I. (2019). The Blockchain Challenge for Higher Education Institutions. European Journal of Education, 2(3), 41. https://doi.org/10.26417/ejed.v2i3.p41-46

Housley, R. (2004). Public Key Infrastructure(PKI). The Internet Encyclopedia. https://doi.org/10.1002/047148296x tie149

Iredale, G. (2021). 6 key blockchain features you need to know now. 101 Blockchains. Retrieved September 27, 2021, from https://101blockchains.com/introduction-to-blockchain-features/.

Liang, X., \& Zhao, Q. (2020). On the design of a blockchain-based Student Quality Assessment System. 2020 International Conference on High Performance Big Data and Intelligent Systems (HPBD\&IS). https://doi. org/10.1109/hpbdis49115.2020.913058

Loukil, F., Abed, M., \& Boukadi, K. (2021). Blockchain adoption in education: A systematic literature review. Education and Information Technologies, 26(5), 5779-5797. https://doi.org/10.1007/s10639-021-10481-8

Ma, Y., \& Fang, Y. (2020). Current status, issues, and challenges of blockchain applications in education. International Journal of Emerging Technologies in Learning (IJET), 15(12), 20. https://doi.org/10.3991/ijet. v15i12.13797 
Magazine, B. (2015). Beyond bitcoin: How the blockchain can power a new generation of enterprise software. Bitcoin Magazine: Bitcoin News, Articles, Charts, and Guides. https://bitcoinmagazine.com/business/ beyond-bitcoin-how-the-blockchain-can-power-a-new-generation-of-enterprise-software-1443635470.

Malavolta, G., Moreno-Sanchez, P., Schneidewind, C., Kate, A., \& Maffei, M. (2019). Anonymous multi-hop locks for Blockchain scalability and interoperability. Proceedings 2019 Network and Distributed System Security Symposium. https://doi.org/10.14722/ndss.2019.23330

Mingxiao, D., Xiaofeng, M., Zhe, Z., Xiangwei, W., \& Qijun, C. (2017). A review on consensus algorithm of blockchain. 2017 IEEE International Conference on Systems, Man, and Cybernetics (SMC). https://doi. org $/ 10.1109 / \mathrm{smc} .2017 .8123011$

Nakamoto, S. (2009). Bitcoin: A peer-to-peer electronic cash system. Retrieved September 28, 2021.

NuWire. (2021). The role of Blockchain technology in the education sector. NuWireInvestor. Retrieved September 30, 2021, from https://www.nuwireinvestor.com/the-role-of-blockchain-technology-in-the-education-sector/

Office of Educational Technology (2021). Blockchain in education https://tech.ed.gov/blockchain/.

Petersen, K., Feldt, R., Mujtaba, S., Mattsson, M. (2008). Systematic mapping studies in software engineering. In 12th International Conference on Evaluation and Assessment in Software Engineering (EASE) 12 (pp. 1-10).

PixelPlex. (2021). Blockchain education applications \& use cases. PixelPlex. Retrieved September 28, 2021, from https://pixelplex.io/blog/blockchain-education-use-cases-and-applications/.

Raman, R. K., \& Varshney, L. R. (2018). Distributed storage meets secret sharing on the blockchain. 2018 Information Theory and Applications Workshop (ITA). https://doi.org/10.1109/ita.2018.8503089

Sharma, A. (2018). Blockchain could revolutionize Education next. Here's how. Hacker Noon. https://hackernoon com/blockchain-could-revolutionize-education-next-heres-how-1b720bdf5945

Sharples, M., \& Domingue, J. (2016). The Blockchain and kudos: A distributed system for educational record, reputation and reward. Adaptive and Adaptable Learning, 490-496. https://doi.org/10.1007/978-3-31945153-4_48

SGE. (n.d.). Sony global Education develops technology using blockchain for open sharing of ACADEMIC proficiency and Progress Records. Global Brands Magazine. Retrieved September 28, 2021, from https:// www.globalbrandsmagazine.com/sony-global-education-develops-technology-using-blockchain-for-opensharing-of-academic-proficiency-and-progress-records/.

Swan, M. (2015). Blockchain: Blueprint for a new economy. O'Reilly.

Tuson, M. (2018). Gilgamesh platform knowledge - sharing social network platform. Medium. Retrieved September 28, 2021, from https://medium.com/@kotpodkolpakom/gilgamesh-platform-knowledge-sharingsocial-network-platform-178c68bfae6d.

Ullah, N., Mugahed Al-Rahmi, W., Alzahrani, A. I., Alfarraj, O., \& Alblehai, F. M. (2021). Blockchain technology adoption in Smart Learning Environments. Sustainability, 13(4), 1801. https://doi.org/10.3390/su13041801

Yli-Huumo, J., Ko, D., Choi, S., Park, S., \& Smolander, K. (2016). Where is current research on blockchain technology? - a systematic review. PLOS ONE, 11(10). https://doi.org/10.1371/journal.pone.0163477

Zheng, Z., Xie, S., Dai, H., Chen, X., \& Wang, H. (2017). An overview of blockchain technology: Architecture, consensus, and future trends. 2017 IEEE International Congress on Big Data (BigData Congress). https://doi. org/10.1109/bigdatacongress.2017.85 